Internal Report

DESY M-89-25

December 1989

Status and Future Developments of the Wake Field Transformer Experiment

N. Holtkamp, P. Schütt, R. Wanzenberg, T. Weiland Technische Hochschule Darmstadt

W. Bialowons, M. Bieler, F.-J. Decker, H.-C. Lewin Deutsches Elektronen-Synchrotron DESY, Hamburg 


\title{
Status and Future Developments of the Wake Field Transformer Experiment
}

\author{
N. Holtkamp, P. Schütt, R. Wanzenberg, T. Weiland \\ Technische Hochschule Darmstadt \\ Fachgebiet Theorie Elektromagnetischer Felder \\ Schloßgartenstr. 8, 6100 Darmstadt \\ W. Bialowons, M. Bieler, F.-J. Decker, H.-C. Lewin \\ Deutsches Elektronen-Synchrotron DESY \\ Notkestr. 85, 2000 Hamburg 52, Germany
}

contributed to:

Second All-Union Workshop New Methods of Particle Acceleration

Nor Ambard, October 10. - 16. , 1989 (Yerewan, USSR) 


\title{
Status and Future Developments of the Wake Field Transformer Experiment
}

\author{
N. Holtkamp, P.Schütt, R. Wanzenberg, T. Weiland \\ Technische Hochschule Darmstadt \\ Fachgebiet Theorie Elektromagnetischer Felder \\ Schloßgartenstr. 8, 6100 Darmstadt \\ W. Bialowons, M. Bieler, F.-J. Decker, H.-C. Lewin \\ Deutsches Elektronen-Synchrotron DESY \\ Notkestr. 85, 2000 Hamburg 52, Germany
}

\section{Introduction}

Linear colliders with high accelerating gradients are the most promising way to reach $\mathrm{e}^{+} \mathrm{e}^{-}$reactions with an energy above $1 \mathrm{TeV}$. The necessary high accelerating-gradients can be provided by Wake Field Transformers [1]. In a Wake Field Transformer a highly charged driving beam excites electromagnetic waves, which are spatially focused in a strong electric field, to accelerate a second beam. In this paper the most recent results of the experiments with a $20 \mathrm{~cm}$ long transformer section are given.

The second part gives an overview of present investigations on a new Resonant Wake Field Transformer experiment. Calculations concerning the energy transfer and efficiency of a multibunch driver sheme are presented. Also new ideas for a hollow beam gun to produce current pulses of nearly $100 \mathrm{nsec}$ are considered. Furthermore, a new scheme is introduced, which allows strong focussing of hollow beams.

\subsection{Basic Idea}

The idea of Wake Field Acceleration originates from the observation that wake fields in existing accelerators can produce enormously strong decelerating and deflecting wake potentials. However, the wake potential for particles at the tail is positive (acceleration). For short symmetric bunches the maximum accelerating potential behind the bunch cannot exceed twice the maximum decelerating potential inside the bunch. The situation is significantly improved using the mechanism of Wake Field Transformation [1]. Two beams, a high charge driving beam of $1 \mu \mathrm{C}$, (e.g. a hollow beam), and a low charge driven beam of $0.01 \mu \mathrm{C}$ traverse, at different locations, a special kind of a multi-cell cavity which we call a Wake Field Transformer. In this transformer the driving beam excites wake fields that lead to its deceleration. By properly shaping the transformer geometry, the driving beam can excite a wave packet that is spatially focused. Thus, there is a, increase in field strength proportional to the inverse square root of the volume containing the wake fields. A second pulse of particles, entering the transformer on axis with proper delay, experiences this concentrated wake field and thus an acceleration, which is much greater than the deceleration of the driving beam.

This scheme is considered to be a candidate for the next $\mathrm{TeV}$ electron-positron collider, because accelerating gradients will exceed $100 \mathrm{MeV}$ per meter. It could be built within a total length of 
about $10 \mathrm{~km}$ if the predicted gradients can be reached. An experiment with a hollow beam Wake Field Transformer has been set up at DESY.

\subsection{Overall Experimental Set Up of Stage II}

In order to study the problems associated with the Wake Field Transformer concept we have mounted an experiment using a complete linac to generate the driving beam. We have chosen a cylindrically symmetric transformer since it should provide high transformation ratios and since it was the only one that could properly be analyzed when first proposed in the year 1982 . With a stage-I experiment at DESY, which is described in another paper, a gradient of $8 \mathrm{MeV} / \mathrm{m}$ in a 2-cell transformer has been achieved [2]. Here we present the stage II results . The driving hollow beam has a diameter of $10 \mathrm{~cm}$. When extracted from the gun the charge should be $1 \mu \mathrm{C}$ over a pulse length of $1 \mathrm{~ns}$. Thus, we need $1 \mathrm{kA}$ electron current. The design peak value of the pulsed cathode voltage is $150 \mathrm{kV}$. The beam is accelerated to about $7 \mathrm{MeV}$ and compressed before entering the Wake Field Transformer. The overall set up of the experiment is shown in Fig. 1.

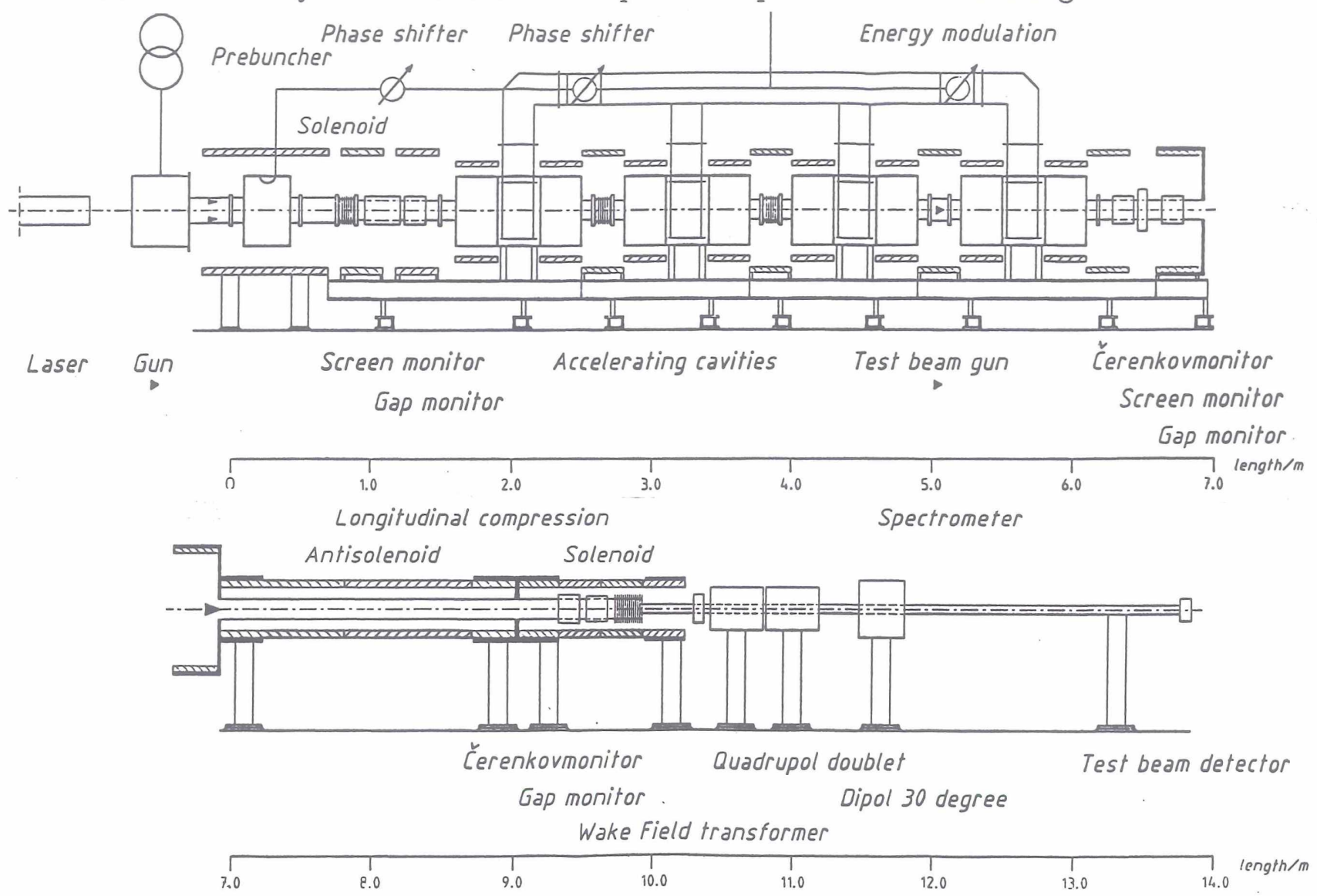

Figure 1: Stage II set up of the Wake Field Transformer experiment at DESY.

Shown from left to right: $A$ laser driven electron gun produces a hollow beam of diameter $10 \mathrm{~cm}$ at an energy of about $150 \mathrm{keV}$. The beam is guided by solenoid fields of $0.2 \mathrm{~T}$ along the linac. First, it is, compressed longitudinally in a prebuncher. Then, four 3 -cell cavities ( $500 \mathrm{MHz})$ accelerate it to about $7 \mathrm{MeV}$. In the antisolenoid further longitudinal compression is achieved. The energy of the central witness beam passing through this transformer on axis is analyzed in a spectrometer adjacent to the transformer. 


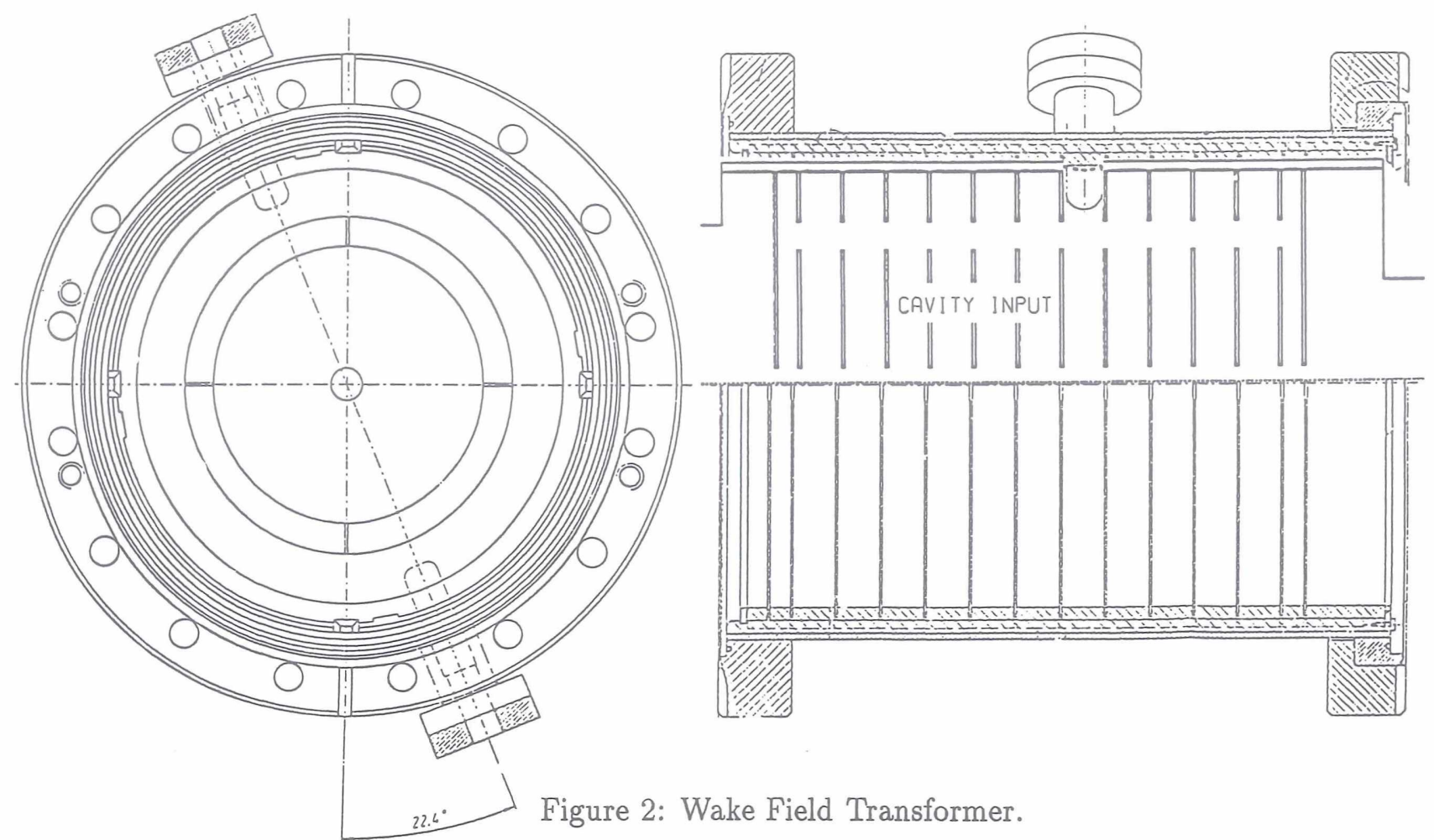

The section consists of $12(11+2 / 2)$ transformer cells. Two glass domes are included for coupling loops. In the upper half of the right part the input shape for numeric calculations is put into the technical drawing, to see the slots for the hollow and the test beam respectively.

\section{New Assembly with a Resonant Transformer}

To study a longer transformer section a relativistic test beam and a corresponding energy spectrometer are needed. The space of $8 \mathrm{~cm}$ between the $3^{\text {rd }}$ and $4^{\text {th }}$ cavity (compare Fig. 1 ) was enough to install a field emission gun, including a small bellow and the flanges. Behind the $4^{\text {th }}$ cavity the central test beam must go exactly through the middle of the solenoid-antisolenoid section to avoid the excitation of helical motion. After passing the Wake Field Transformer the beam leaves the solenoid field and enters a spectrometer, which consists of two quadrupoles, and one $30^{\circ}$ dipole. Here, the particles are focussed onto a screen, which is observed by a photomultiplier, to measure the intensity with respect to the momentum.

The design of the Resonant Wake Field Transformer has to fulfill several constraints for our experiment. First the resonance frequency must be generally a multiple of the driving frequency, in our case a frequency ratio of $\nu / 500 \mathrm{MHz}=8$. Second for a given hollow beam radius the outer boundary of the transformer cavity was chosen in such a way, that the decelerating field at the radius of the beam has a local maximum. This provides a strong coupling of the drive beam to this mode at the expense of a low transformation ratio. However, the idea was to study a longer transformer section rather than to achieve a high gradient.

In the transformer (compare Fig. 2) two glass domes were included to get a reference signal. The first and the last cell are only half cells. The slot for the hollow beam was electromagnetically closed by a grid at the entrance and the exit. With $11+2 / 2$ cells we get a $3 \pi / 12$ mode, which has the right phase advance, phase velocity and frequency to get a special closed transformer cavity which could be tested in an RF-laboratory; a resonant frequency of $4.01 \mathrm{GHz}$ for the selected mode was measured [3]. 


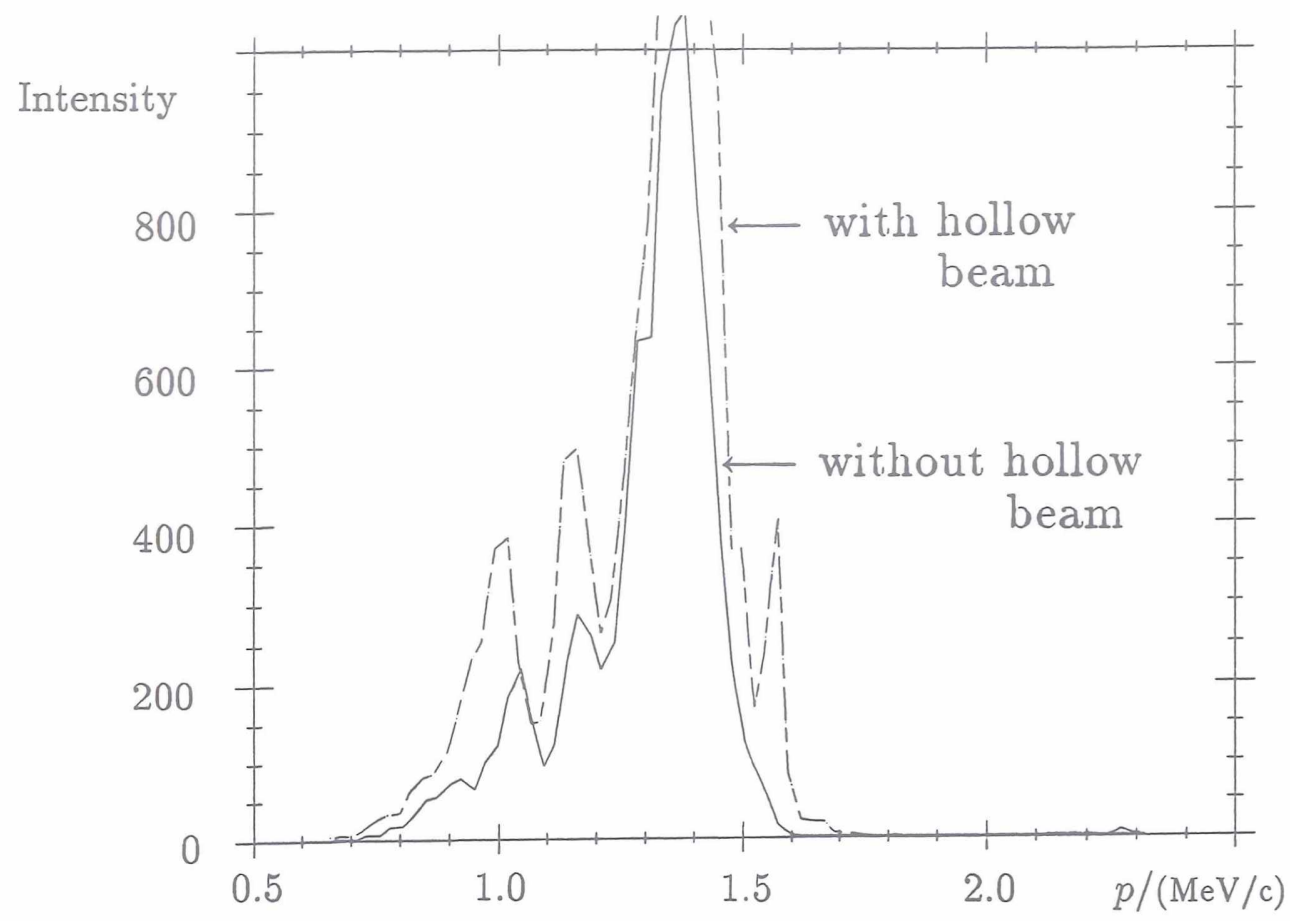

Figure 3: Wake field acceleration.

With the hollow beam exciting the transformer, higher energies were observed. The gun current of the hollow beam was only $6 \mathrm{~A}$ for which a gradient of $1.2 \mathrm{MeV} / \mathrm{m}$ was expected. Broadening of the energy distribution by $100 \mathrm{keV}$ means a gradient of $1.2 \mathrm{MeV} / \mathrm{m}$ for the $16.2 \mathrm{~cm}$ long transformer section.

\section{Commissioning and Measurement}

The three major parts of the new set up, test beam gun, $20 \mathrm{~cm}$ transformer and spectrometer were commissioned nearly simultaneously. The energy resolution of the set up was calibrated by reducing the voltage of the $4^{\text {th }}$ cavity by three percent. This corresponds to a momentum reduction of $37 \mathrm{keV} / c$, which could easily be resolved by the spectrometer. In our Wake Field Transformer a momentum change of $33 \mathrm{keV} / \mathrm{c}$ would be equivalent to a gradient of $150 \mathrm{keV} / \mathrm{m}$, therefore it should be possible to observe even small energy variations. Different possibilities have been tried to achieve and observe this, but in the first experiments we measured a decrease in energy. With the hollow beam exciting the transformer, we have also beam loading induced in the fourth cavity and the energy of the test beam is lowered. Therefore we scraped the the hollow beam in front of the transformer (and after the cavities) by a vacuum valve which had a hole in its center. In this case the intensity peak in the energy distribution of the test beam was broadened as it was predicted (see Fig. 3). The agreement with the expected accelerating gradient of about $1.2 \mathrm{MeV} / \mathrm{m}$ is quite good in the region of low hollow beam currents between 5 and $10 \mathrm{~A}$. However at higher currents (30-40 A) nearly the same acceleration was observed. We found that the distance between the hollow beam bunches was elongated. Decreasing energy from bunch to bunch due to beam loading is transformed into a distance variation in the high energy buncher (compare Fig. 1) and therefore the resonant excitation of the transformer gets out of phase. Nevertheless the expected $4 \mathrm{GHz}$ oscillation of the transformer, measured with the aerial loop, could be resolved (see Fig. 4), but we found no resonant addition of the fields at higher currents. 

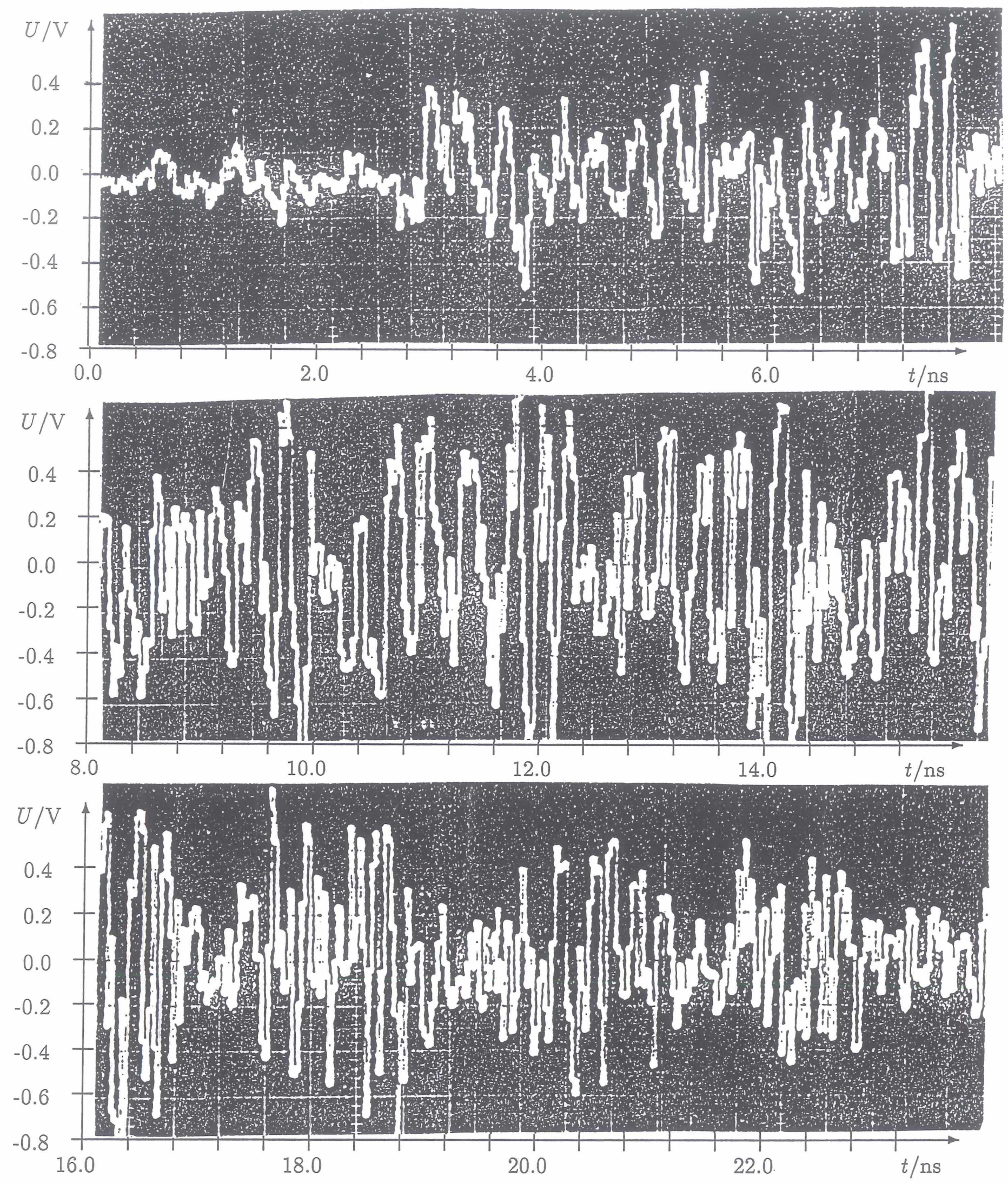

Figure 4: $4 \mathrm{GHz}$ resonance in the Wake Field Transformer.

The voltage response of the aerial loop mounted in the wake field transformer is shown. Five to six hollow beam bunches excite the transformer with a time distance of $2 \mathrm{~ns}$. Between two succeeding bunches one can observe an oscillation of $4 \mathrm{GHz}$ ( 8 peaks). It can be seen that the expected summing up of all bunch excitations does not happen. 

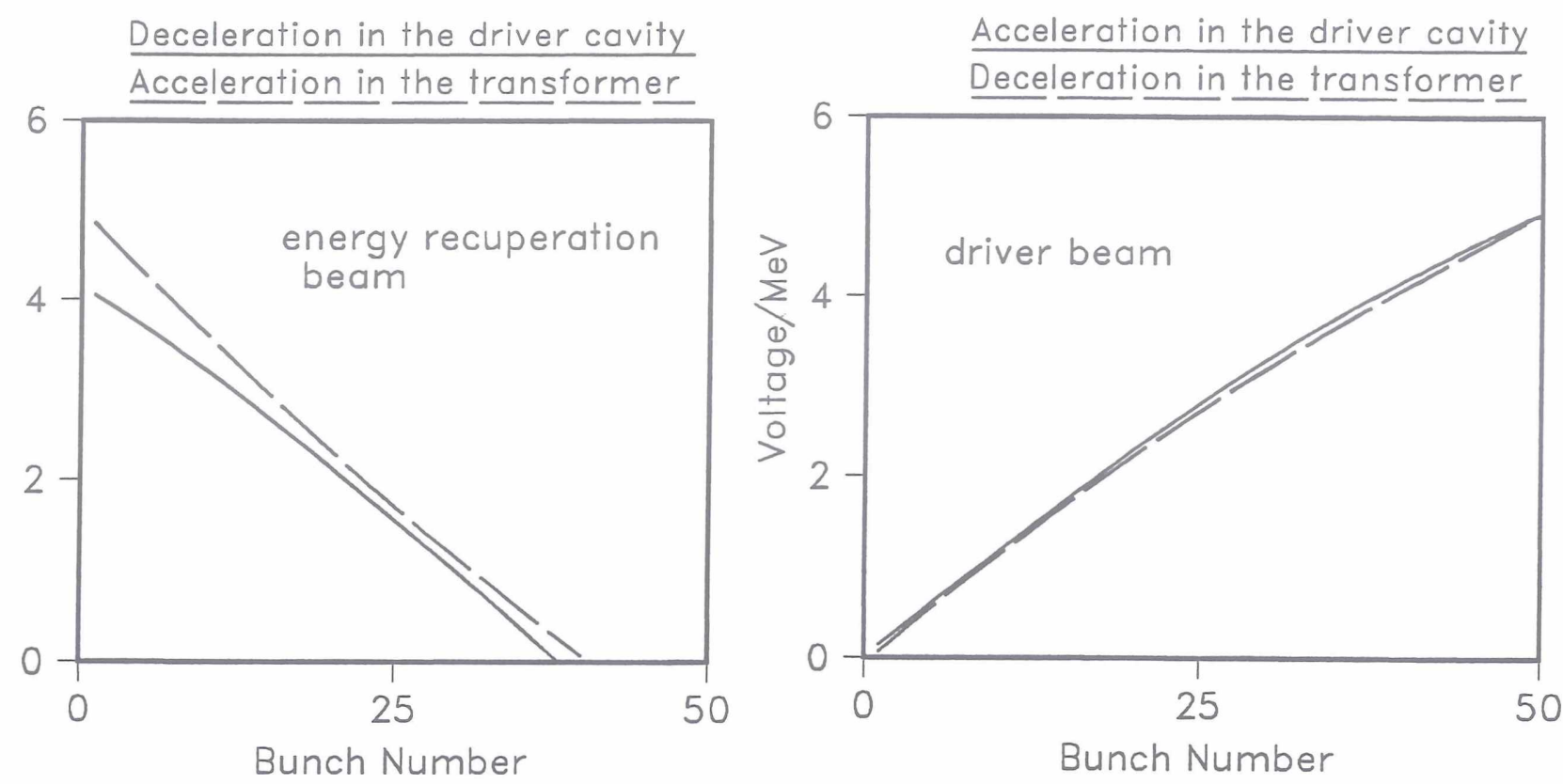

Figure 5: Voltage in the driver cavities and the transformer as a function of bunch number.

The voltage in the driver cavities is varied from bunch to bunch (solid line) to produce an energy spread which is compensated in the transformer due to beam loading induced by the preceeding bunches. This decelerating voltage is raised up nearly linear (dashed line).

\section{Further Research and Development for a Multibunch Dri- ver Scheme}

The next generation of a Wake Field Transformer experiment has to fulfill several conditions, which could not be reached with the set up shown in Fig. (1). The decision to use a multibunch driver beam leads to a completely new concept of efficiency and energy transfer to accelerate the test beam. In this linac a special longitudinal dynamic is chosen in order to realize this optimum. Two trains of hollow beam bunches are used to load the transformer and also to recuperate the energy left by the central beam. Some of the ongoing calculations for the envisiged TeV-Collider as well as for the proposed test experiment are presented. Nevertheless, the various components, for example, the gun or the hollow beam lenses, have to be tested.

\subsection{New Concepts for the Resonant Wake Field Transformer Experiment}

From the experimental results it was evident, that the production and handling of hollow beam bunches with very high charge $(\geq 1 \mu \mathrm{C})$ especially in the low energy part of the linac is very complicated. Dividing the charge into several bunches and using a kind of transformer cavity for the resonant addition of the excited Wake Fields, makes it possible to reach the same overall gradient along the linac [4]. The bunches enter the cavities at an increasing cavity voltage, in order to compensate the beam loading in the transformer, which grows from bunch to bunch (Fig 5).

The accelerating voltage, seen by the $\mathrm{n}$ - th bunch in a $500 \mathrm{MHz}$ driver cavity,

$$
V_{1}(n)=L_{1} V_{0}^{\prime} \cos \left(\phi_{0}+n \Delta \phi\right)-2 q_{t o t} k_{1}^{\prime} \frac{1}{N}\left(\frac{1}{2}+\sum_{m=1}^{n-1} \cos (m \Delta \phi)\right)
$$

and the corresponding decelerating voltage in the transformer

$$
V_{2}(n)=-L_{2} 2 q_{t o t}\left(k_{2}^{\prime} / \mathrm{t}^{2}\right) \frac{1}{N}\left(\frac{1}{2}+\sum_{m=1}^{n-1} \exp \left(-2 \pi h m / 2 Q_{2}\right)\right)
$$




\begin{tabular}{|c|c|c|c|}
\hline \multicolumn{4}{|c|}{ cavity parameters } \\
\hline & driver & & transformer \\
\hline$f_{1}$ & $500 \mathrm{Mhz}$ & $f_{2}$ & $12 \mathrm{GHz}$ \\
\hline$Q_{1}$ & $310^{9}$ & $Q_{2}$ & 8100 \\
\hline$k_{1}^{\prime}$ & $0.32 \mathrm{~V} /(\mathrm{m} \mathrm{pC})$ & $k_{2}^{\prime}$ & $213.0 \mathrm{~V} /(\mathrm{m} \mathrm{pC})$ \\
\hline & & on axis & \\
\hline$V_{0}^{\prime}$ & $10 \mathrm{MV} / \mathrm{m}$ & $\mathrm{t}$ & 35 \\
\hline \multicolumn{4}{|c|}{ bunch parameters } \\
\hline \multicolumn{2}{|c|}{ first beam } & \multicolumn{2}{|c|}{ recuperation beam } \\
\hline$N_{1}$ & 50 & & 40 \\
\hline$q_{\text {bun } 1}$ & $122 \mathrm{nC}$ & $q_{\text {bun } 2}$ & $100 \mathrm{nC}$ \\
\hline$\Phi_{1}$ & $55^{\circ}$ & $\Phi_{2}$ & $44^{\circ}$ \\
\hline \multicolumn{4}{|c|}{ gradient,length ratio and efficiencies } \\
\hline $\begin{array}{l}G_{\text {ave }} \\
L_{2} / L_{1}\end{array}$ & $\begin{array}{c}45 \mathrm{MV} / \mathrm{m} \\
2.9\end{array}$ & \multicolumn{2}{|c|}{$\begin{array}{l}\text { Average Gradient } \\
\text { length ratio }\end{array}$} \\
\hline \multicolumn{4}{|c|}{ efficiencies } \\
\hline$\eta_{1}$ & $71 \%$ & \multirow{3}{*}{\multicolumn{2}{|c|}{$\begin{array}{l}\text { hollow beam to transformer } \\
\text { transformer to recuperation beam } \\
\text { recuperation beam to driver }\end{array}$}} \\
\hline$\eta_{2}$ & $78 \%$ & & \\
\hline$\eta_{3}$ & $86 \%$ & & \\
\hline
\end{tabular}

Table 1: Results for a $500 \mathrm{GHz}$ driver cavity

can be varied to give an optimum in efficiency. The important design parameters are:

$\begin{array}{ll}V_{0}^{\prime} & \text { the driver cavity voltages per meter } \\ q_{\text {tot }} & \text { the total charge in the train of } N \text { bunches } \\ \Phi=N \Delta \phi & \text { the total phase advance } \\ \mathrm{t} & \text { the transformer ratio } \\ h & \text { the harmonic number between driver and transformer frequency } \\ g=1 /\left(1-\eta_{1} \eta_{2} \eta_{3}\right) & \text { the gain } ;=1 \text { without energy recuperation }\end{array}$

Note that the loss parameter per meter $k^{\prime}=\omega R^{\prime} /(2 Q)$ scales with $h^{2}$. Some numerical results for a possible optimized set up of a $500 \mathrm{MHz}$ driver section with length $\mathrm{L}_{1}$ and a corresponding $12 \mathrm{GHz}$ transformer cavity with length $\mathrm{L}_{2}$ are presented in table (1).

Having reached the maximum voltage on axis, the central beam passes the loaded transformer. But only a few percent of the stored energy can be used, because otherwise the energy spread of the test beam becomes too large. Before loosing the energy into wall losses, a second train of hollow beam bunches passes the transformer with a phase shifted by 180 degree, in order to transport it into the following superconducting cavity where energy loss can be neglected due to the high $\mathrm{Q}$-values. In this way an energy recuperation scheme is included, which reduces klystron power, because all but the first of the cavities, running in cw-mode, are repowered by a second driver beam. It follows from this principle, that a very accurate timing of the gun and the buncher section is needed, but the moderate frequency of $500 \mathrm{MHz}$ in the driver linac should make this possible. The efficiency $\eta_{1}$ is limited by conversion of energy $V_{1}(n) \geq V_{2}(n) n=1 \ldots N$. Analytical calculations show that

$$
\eta_{1} \leq \frac{\Phi_{1}}{2 \tan \Phi_{1} / 2}
$$

The theoretical limit is not reached due to wall losses in the transformer included the above numerical calculations. Further optimisations are expected in the ongoing calculations. 


\subsection{The Hollow Beam Gun}

From table (1) we can see, that 90 bunches are needed with a charge of $100 \mathrm{nC}$ per bunch. Therefore the gun must provide a current pulse with a length of at least $180 \mathrm{nsec}$ and a current of 50 Ampere. Up to now two possibilities are investigated to reach long current pulses with a hollow beam gun. The pulse of a laser, which illuminates the cathode of the hollow beam gun, is splitted and half of its energy stored in a Photon Storage Ring. The intensity of the outgoing pulse decreases nearly
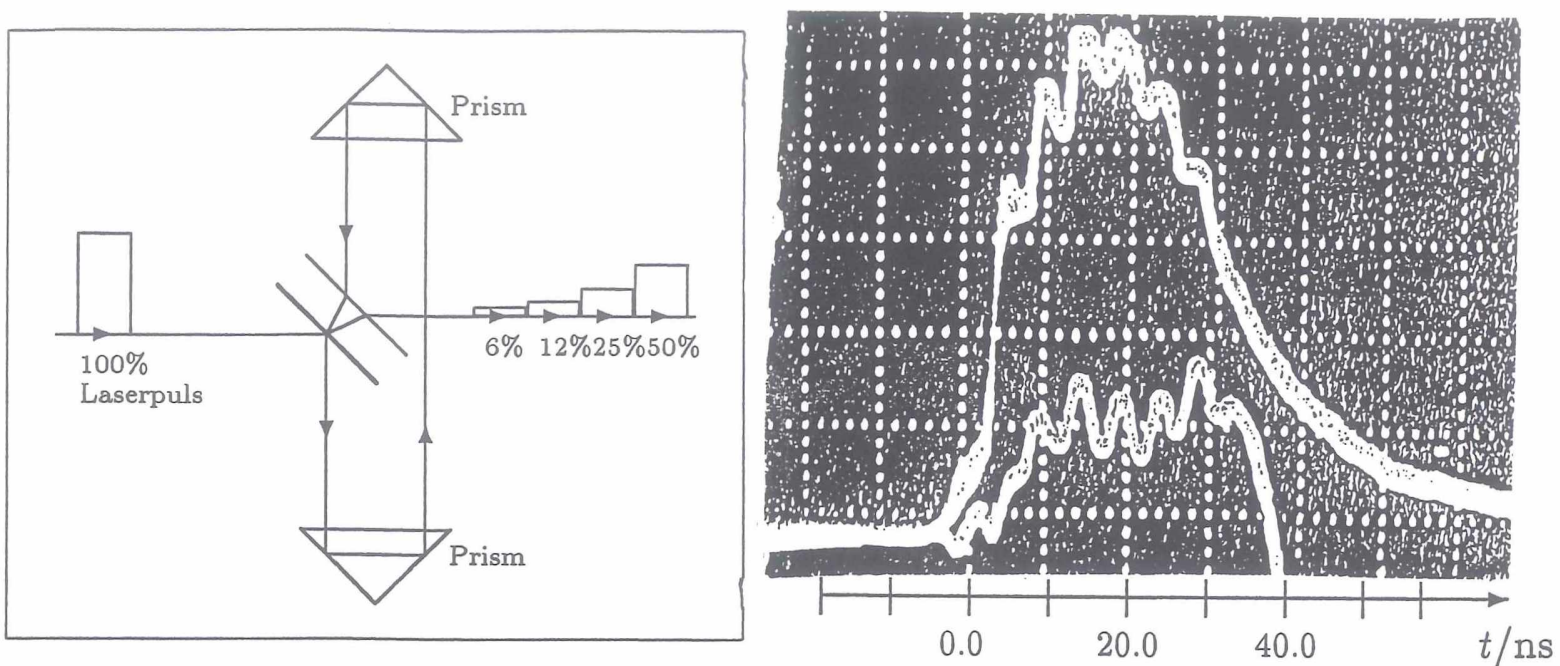

Figure 6: Principle of a Photon Storage Ring

The Light pulse of a laser is splitted and some part of the energy is delayed. The light is reflected by two prisms and outcoupling of the energy is performed by a semipermeable mirror. The original pulse length of $10 \mathrm{nsec}$ is broadened to at least $30 \mathrm{nsec}$.

quadratically, if the cicumference of the storage ring devided by $\mathrm{c}$ is equal to the original length of the light pulse. Figure (6) shows a shematic view of the storage ring and the corresponding measured beam current with an ingoing pulse length of $10 \mathrm{nsec}$.

Another possibility is the use of thin layers which are evaporated on a cathode. For example, a metallic surface is first coated with $\mathrm{Al}_{2} \mathrm{O}_{3}$ for thermal isolation and then again evaporated with a $150 \mathrm{Im}$ thick tantalum layer. If the layer is illuminated by a laser pulse, thermal diffusion of the deposited energy can only take place within the tantalum surface and not back into the metal. The thermal response of the tantalum is fast compared to the length of the laser pulse, but compensation will take much more time depending on the thickness of the layer. This corresponds to a delayed thermionic emission of electrons and therefore a longer current pulse from the surface. First measurements have shown, that pulses longer than $30 \mathrm{~ns}$ could be reached by both methods. Combining the methods will further improve these results.

\subsection{A New Strong Focussing Scheme for Hollow Beams}

Up to now, the whole linac was mounted within solenoid coils (see Fig. 1) to guide the hollow beam. A concept, using strong focussing, as described for central beams in [6], has the great advantage of field free driftspace and smaller transverse dimensions. Superconducting cavities and long transformer sections can be installed in between the hollow beam lenses. The focussing is provided by permanent magnets which nowadays can have remanent fields of more than 1.5 Tesla. The axially symmetric arrangement of the magnets shown in figure (7), leads to a special solenoid antisolenoid combination, with a field gradient perpendicular to the reference ring with radius $\rho$. Solving the equations of motion in the frame $(x, y, s)$ under the assumption of a thin lens, the net azimuthal rotation vanishes if going through the whole solenoid antisolenoid. This is very important, because the inner magnets could be fixed with four tags at the beginning and the end 
Figure 7: 2-D cut of azimuthal symmetric hollow beam lens.

Permanent magnets with remanent fields of up to 1.5 Tesla can be used to focus hollow beams. The axially symmetric arrangement provides a special soleniod antisolenoid field, which acts like a lens with fokal length of 5 to $10 \mathrm{~m}$ at hollow beam energies of nearly $500 \mathrm{MeV}$.

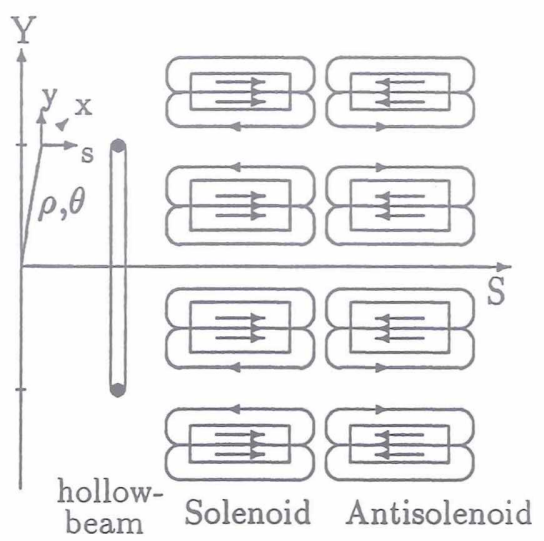

of the lens without loosing a significant portion of electrons. Particles on a seperated path from $\rho$ with $r$ not equal zero are always focussed towards the hollow beam axes with a focussing strength proportional to $\left(\gamma / \mathrm{B}_{z}\right)^{2}$

Therefore only the radial motion has to be regarded. A linac with such kind of magnets consists only of driftspaces and focussing lenses. To determine the trajectories in $r$, a matrix representation of these two linac components in the frame $(r, \theta, s)$ can be calculated, which is completely analogue to the one known from central beams in cartesian coordinates.

$$
\left(\begin{array}{c}
r_{f} \\
r_{f}^{\prime}
\end{array}\right)=\left(\begin{array}{ll}
1 & L \\
0 & 1
\end{array}\right) \cdot\left(\begin{array}{c}
r_{i} \\
r_{i}^{\prime}
\end{array}\right) ;\left(\begin{array}{c}
r_{f} \\
r_{f}^{\prime}
\end{array}\right)=\left(\begin{array}{cc}
1 & 0 \\
-K^{2} l & 1
\end{array}\right) \cdot\left(\begin{array}{c}
r_{i} \\
r_{i}^{\prime}
\end{array}\right)
$$

The meaning of the symbols is:

$$
\begin{array}{ccc}
1 & = & \text { length of the solenoid antisolenoid } \\
\mathrm{K} & = & q \cdot c /(2 \cdot p \cdot c) \cdot \mathrm{B}_{z} \\
\mathrm{~K}^{2} \cdot 1 & = & 1 / \mathrm{f}, \mathrm{f}=\text { focal length }
\end{array}
$$

The anology is also valid for the well known amplitude- and phasefunctions $(\beta(s), \phi(s))$, defined by Courant and Snyder [6] in 1958. The two eigenvectors, calculated from the solution of Hill's differential equation are:

$$
\begin{aligned}
& \left(\begin{array}{l}
r_{1} \\
r_{1}^{\prime}
\end{array}\right)=\left(\begin{array}{l}
\sqrt{\varepsilon \beta} \cdot \cos \left(\phi-\phi_{0}\right) \\
\sqrt{-\varepsilon / \beta} \cdot \sin (\Delta \phi)+\alpha \cos (\Delta \phi)
\end{array}\right) \\
& \left(\begin{array}{c}
r_{2} \\
r_{2}^{\prime}
\end{array}\right)=\left(\begin{array}{l}
\sqrt{\varepsilon \beta} \cdot \sin \left(\phi-\phi_{0}\right) \\
\sqrt{-\varepsilon / \beta} \cdot \cos (\Delta \phi)-\alpha \sin (\Delta \phi)
\end{array}\right)
\end{aligned}
$$

With:

$$
\begin{array}{rlc}
\beta(\mathrm{s}) & = & \text { amplitudefunction in } \mathrm{s} \\
\varepsilon & = & \text { emittance of the holllow beam in } r \\
\alpha(\mathrm{s}) & = & -\frac{1}{2} \cdot \beta(\mathrm{s}) \\
\phi(\mathrm{s}) & = & \phi_{0}+\int_{0}^{s} 1 / \beta \mathrm{dz} \text { phasefunction }
\end{array}
$$

In the case of hollow beams they are given on the beam radius $\rho$ and they have azimuthal symmetry going at a given point s once around the reference ring with radius $\rho$.

\section{Proposal of a New Resonant Wake Field Transformer Linac}

With the concepts, described in the last section, it is possible to calculate the $\beta$-function along a normal cell of a Resonant Wake Field Transformer linac including the lenses and the driftspaces 


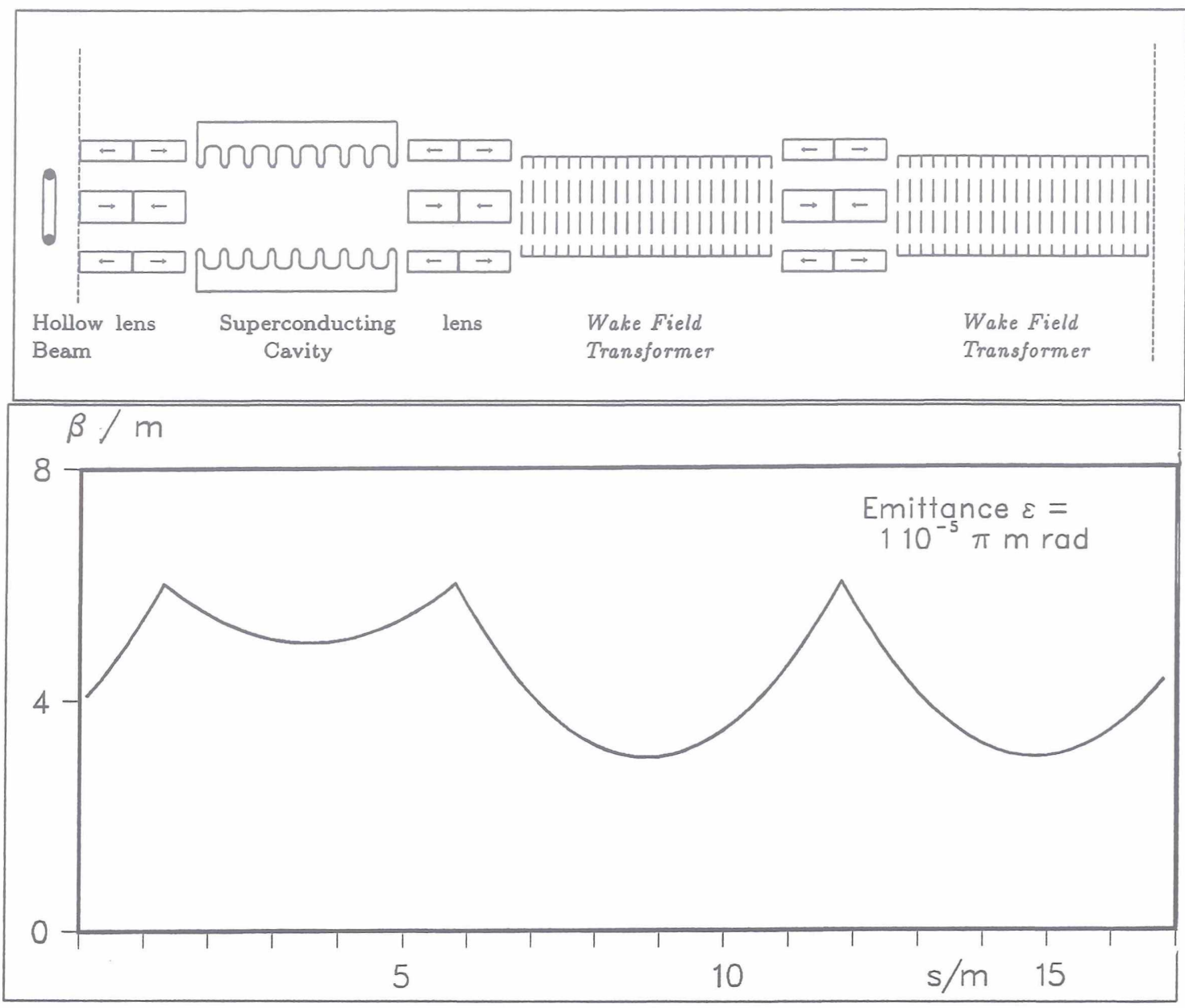

Figure 8: A Normal Cell in the New Resonant Wake Field Transformer Experiment.

The proposed Resonant Wake Field TransformerCollider consists of several normal cells like the one shematically given in the first picture. The shape of the corresponding radial $\beta$-function of the hollow beam is shown below. It has the same symmetry and should have the minima in the transformer, because of the small diameter of the hollow beam slots.

for superconducting cavties and transformers. The linac can be mounted by a series of such normal cells. For the first calculations a hollow beam radius of $5 \mathrm{~cm}$ is given and the half-aperture in the lenses, where the $\beta$-function has its maximum, is taken to be $1 \mathrm{~cm}$. Also in the cavities the hollow beam may have quite large dimensions, whereas in the transformer smaller radial diameters are needed because of the thin slots in the disks. A possible layout for a normal cell is presented in Fig. 8.

This kind of structure is considered for a new test accelerator, to gain the experience for building the $\mathrm{TeV}$ Collider. In comparison with other concepts, the idea of a resonant impedance transformation of Wake Fields seems to have a lot of advantages. For example, the CERN collider CLIC uses a similar principle, but with an ordinary driver beam needing charge densities which are 10-50 times higher. Other serious ideas are not in a stage of possible construction and more classical concepts, using more $(\geq 10000)$ and better klystrons, are unlikely to open the energy range above $1 \mathrm{TeV}$ without an enormous amount of wall plug-power. 


\section{References}

[1] G.-A.Voss and T.Weiland, Particle Acceleration by Wake Fields, DESY M-82-10, April 1982.

[2] W. Bialowons, M. Bieler, H.-D. Bremer, F.-J. Decker, N. Holtkamp, H.-C. Lewin, P. Schütt, G.-A. Voss, R. Wanzenberg, T. Weiland, Wake Field Acceleration - A New Mechanism for Particle Acceleration, DESY 89-037, March 1989.

[3] N. Holtkamp, R. Wanzenberg, T. Weiland, Technische Hochschule Darmstadt, Institut für Hochfrequenztechnik, Fachgebiet Theorie Elektromagnetischer Felder, Schloßgartenstr. 8, 6100 Darmstadt and W.Bialowons, M. Bieler, F.-J. Decker, H.-C. Lewin, M. Nagl, U. Niemeier, Deutsches ElektronenSynchrotron DESY, Notkestr. 85, 2000 Hamburg 52, Germany, Messungen der Moden beim resonanten Wake Field Transformator, DESY, M-89-05, July 1989.

[4] T. Weiland et al, The Resonant Wake Field Transformer Concept for Particle Acceleration. DESY M-87-10, July 1987 and European Particle Accelerator Conference (EPAC), Rome, June 1988, pp. 490.

[5] Wilhelm Bialowons, Hohlstrahlkanone für das Wake Field Transformatorexperiment bei DESY, Thesis, II. Institut für Experimentalphysik der Universität Hamburg, DESY M-87-10, July 1987.

[6] E.D. Courant,H.S. Snyder Theory of Alternating Gradient Synchrotron, Annals of Physics, Vol. 3,No. 1,Jan. 1958 